\title{
Current and Future Respiratory Medicine Challenges: Insights from a Barcelona Respiratory Network Foundation Retreat in Barcelona in 2017
}

Jordi Dorca, MD, PhD ${ }^{1,5}$, Alvar Agustí, MD, PhD, FRCP, FERS $2,4,5$ and Eduard Monsó, MD, PhD $3,4,5$, on behalf of all the participants listed in the Appendix

${ }^{1}$ Pneumology Service, Hospital Universitari de Bellvitge, L'Hospitalet de Llobregat, Barcelona (Spain). Institut de Recerca Biomèdica de Bellvitge (IDIBELL). Universitat de Barcelona; ${ }^{2}$ Pneumology Service, Thorax Institute, Hospital Clinic, Barcelona (Spain), Institute for Medical Investigation August Pi i Sunyer (IDIBAPS), Universitat de Barcelona; ${ }^{3}$ Pneumology Service, Hospital Universitari Parc Taulí, Terrassa, Barcelona (Spain). Universitat Autònoma de Barcelona; ${ }^{4}$ Centre for Biomedical Investigation Network of Respiratory Disease (CIBERES); ${ }^{5}$ Barcelona Respiratory Network (BRN) Foundation, Barcelona, Spain

\section{ABSTRACT}

On a global level, respiratory diseases impose a major worldwide health burden affecting millions of people. Respiratory diseases are not only very common but present a great challenge to human health and a productive economy. Prevention, control and cure of these conditions are among the most cost-effective interventions to be taken within a healthcare system and must be a priority for researchers as well as for health authorities. Today, many novel technologies can potentially improve the diagnosis and control of numerous diseases, and the present challenge is how they can be quickly developed and implemented. This review summarises the main conclusions of a Barcelona Respiratory Network (BRN) Foundation Retreat held last year in Barcelona. The debate was structured around four distinct sections: 1) trends that impact current society; 2) foreseeable changes in the field of respiratory health; 3) challenges for the future of healthcare systems; and, 4) future biomedical research trends in respiratory medicine. (BRN Rev. 2018;4(3):214-27)

Corresponding author: Dr. Jordi Dorca, jodorca@bellvitgehospital.cat

Key words: Challenges. Research. Trends. 


\section{INTRODUCTION}

Worldwide respiratory diseases constitute a significant global health burden and affect millions of people. Asthma is the most prevalent respiratory disease and according to recent data it affects 358 million people worldwide and causes 400,000 deaths yearly ${ }^{1}$. Chronic obstructive pulmonary disease (COPD) affected 174 million people worldwide in 2015 and is now considered the third most common cause of death worldwide causing 3.2 million deaths per year, representing an increase of $11.6 \%$ compared with $1990^{2}$.

In addition, approximately 65 million display different kinds of occupational lung diseases, and 8.7 million people develop tuberculosis (TB) annually, whereas the prevalence of sleep apnoea ranges between 1 and $6 \%$ of adult population depending on the degree of regional economic development ${ }^{3}$. Finally, there is a wide variety of less prevalent respiratory diseases, including interstitial lung diseases, pulmonary hypertension, and bronchiectasis, among others, which also have marked impact on health status and prognosis of patients with these conditions. According to these figures more than one billion people worldwide suffer from respiratory diseases and about four million people die annually because of these conditions.

Present and future challenges of respiratory medicine were discussed in 2017 during a half-day retreat organised by the Barcelona Respiratory Network (BRN) Foundation, a non-profit foundation aimed to foster research in respiratory medicine. Twenty-two representatives of academic and industrial institutions (listed in the Appendix) attended to openly discuss current and future challenges faced by respiratory medicine.

The debate was structured into four sections: 1) trends that impact current society; 2) foreseeable changes in the field of respiratory health; 3) challenges for the future of health-care systems; and, 4) future biomedical research trends in respiratory medicine. This review summarises the discussions held there.

\section{TRENDS THAT IMPACT CURRENT SOCIETY}

In recent decades there has been an accelerated change within a range of factors which directly and indirectly affect living conditions for modern society. These changes have to do with environmental conditions, the demographic structure of diverse societies, the way these societies organise the economic activity, dietary and toxicological habits of their inhabitants, the impact of modern information and communication technology (ICT) and also a set of values which has been adopted, to a greater or lesser extent, by large percentages of the population. All these circumstances, listed in table 1, will considerably modify dynamics of our different societies on a global level.

There is an accelerated increase in the temperature of the planet derived from massive utilisation of fossil fuels. This will substantially modify the composition of the planet's ecosystems with desertification of extensive tropical zones and the substitution of numerous plant and animal species in the temperate and polar zones. Due in part to this phenomenon and in part to economic 
TABLE 1. Trends that impact modern society

- Climate change

- Tendency of the population to concentrate in large urban centres

- Environmental deterioration.

- Sedentary lifestyle and poor dietary habits

- Obesity

- Changes in tobacco use

- Ageing population leading to chronic diseases

- Great ease of traveling that favours rapid dissemination of new infectious agents

- Impact of information and communication technology (ICT) development: immediate and widespread disclosure of information (truthful and fraudulent), mobile telephony, big data, remote monitoring

- Social aspiration to enjoy a better quality of life

- Citizens more active, organised, better informed and willing to take an active role in healthcare decision-making

- Increasing importance of certain values in society: Responsible Research \& Innovation (RRI) development, the global population is undergoing a process of accelerated urban concentration, which equally affects wealthy and developing countries, with the creation of large mega-cities where economic activity is concentrated (Fig. 1). This generates high levels of environmental pollution having a very direct impact on the health of those inhabitants.

Life habits of individuals in modern society have also notably been altered. Thanks to better social conditions, life expectancy has lengthened considerably. This generates an ageing population in which an increasing percentage of inhabitants suffer from one or several chronic diseases. Furthermore, for the population as a whole, there is a tendency towards sedentary lifestyle and poorer dietary habits which favoured the development of obesity, having reached epidemic proportions in advanced societies (Fig. 2). Certain pervasive habits which have great impact on health, such as tobacco use, have undergone important changes according to the type of

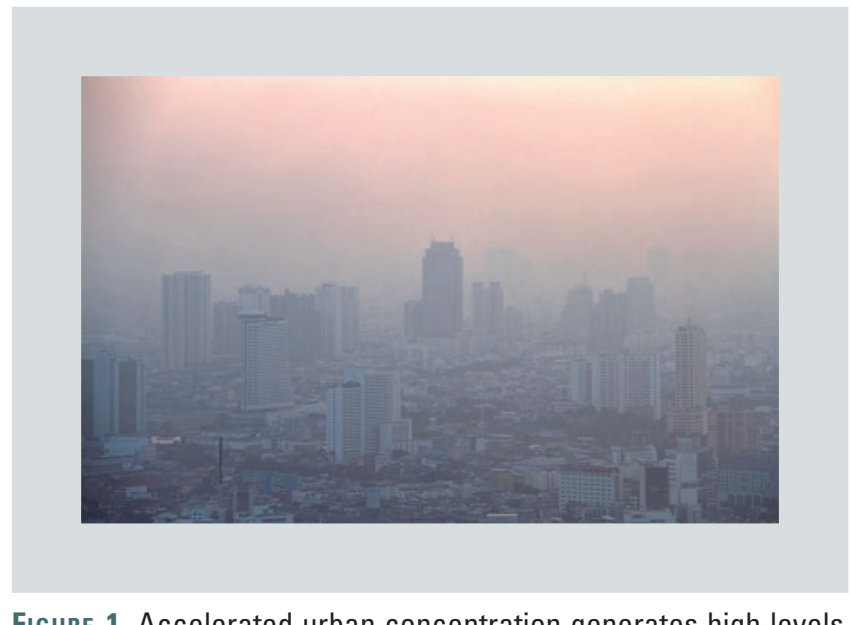

Figure 1. Accelerated urban concentration generates high levels of environmental pollution which has a very direct impact on respiratory health.

society analysed: in developed countries one can generally observe a notable decline in percentage of smokers, while in emerging economies (China, India) tobacco use is growing rapidly, reaching addiction percentages never seen before. Another characteristic of modern society is the great ease of traveling long distances which, among other consequences, facilitates the rapid global diffusion of new and known pathogens, particularly those which are airborne.

The development of modern ICTs derived from digitalisation has given way to radical changes in social organisation, permitting the immediate and generalised broadcast at a global level of any piece of information (be it truthful or fraudulent), the possibility of accessing huge amounts of information (big data), and remote control in real time of a large variety of processes. On the other hand, interpersonal connectivity through mobile devices has reached a worldwide diffusion which has permeated broad sectors of poor countries as well. 


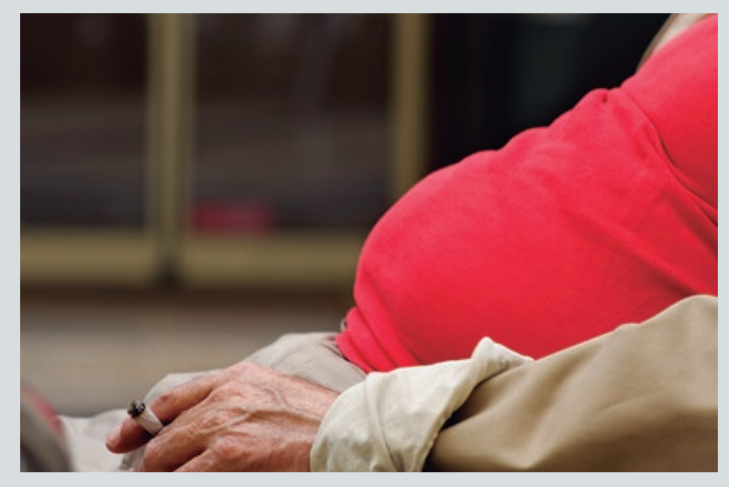

FIgURE 2. Sedentary lifestyle, smoking, poorer dietary habits and obesity have a big impact on health.

These developments have a considerable impact on the way citizens of modern societies live and behave, the way they approach their health problems, as well as on the values that sustain them. In today's society there is a general aspiration to enjoy a better quality of life, to be better informed, to be able to organise collectively and to participate both individually and collectively in making decisions about aspects of life that directly affect a person, including those that have to do with individual health. This includes an ability to participate in deciding priorities during development and planning of research in social and health issues (namely, Responsible Research \& Innovation [RRI]).

\section{FORESEEABLE CHANGES IN THE FIELD OF RESPIRATORY HEALTH}

The changes described above have an impact on human health as well as on the way in which health systems and individuals will respond to health risks. Most of these foreseeable impacts are listed in table 2. Considering the specific case of respiratory medicine, all these phenomena
TABLE 2. Foreseeable changes in the field of respiratory health

- Increase in pathologies related to environmental changes: allergies, asthma, cancer

- Increase in respiratory pathologies related to obesity

- Increased percentage of population that presents with chronic respiratory diseases

- Ease of rapid global spread of infectious airborne pathogens (both new and known)

- Increase in respiratory infections produced by multi drug-resistant bacteria

result in a higher incidence of pathologies related to environmental changes, in particular the deterioration of air quality. Thus, it is expected that incidence of pathologies such as allergies, bronchial asthma or lung cancer will see a rise, well into the future. The increase in the prevalence of obesity is also translating into a higher incidence of metabolic, cardiovascular, skeletal and respiratory diseases, especially those related to nocturnal breathing and bronchial asthma. In fact, these chronic pathologies present multiple interconnections which are commonly associated in the same patient. Given the increase in life expectancy and the progressive ageing of the population, the percentage of individuals suffering from multiple chronic pathologies is increasing, which represents a formidable challenge for health systems.

The ease of traveling long distances has had a great impact on the distribution of certain airborne infections detrimental to respiratory systems, which within a few weeks can spread globally. Recent examples are the severe acute respiratory syndrome (SARS) or Middle East respiratory syndrome (MERS), two zoonoses that after adapting to human hosts rapidly diffused to very distant locations from where they originally emerged. Another numerically relevant example is the 2009 influenza A (H1N1) epidemic that, according to the Centers for 
Disease Control and Prevention (CDC), caused 284,000 deaths worldwide. This dilemma is not only confined to new infectious agents but also to bacterial species against which effective antibiotics were once available but that have now developed multiple resistance and have spread globally as a result of population dynamics. A good example of such a risk would be the case of multi drug-resistant tuberculosis.

\section{CHALLENGES FOR THE FUTURE FOR HEALTHCARE SYSTEMS}

An ageing population naturally leads to the problem of chronic multi-morbidities that affect broad sectors of the population and generate a consequent impact on the healthcare systems in multiple ways, as summarised in table 3.

Chronic respiratory diseases are not only very common, but their prevalence appears to be increasing ${ }^{4}$. In contrast with other major conditions, such as cardiac diseases, stroke and cancer, the mortality rates of which have been decreasing in the last years, death related to COPD has doubled in the same period ${ }^{5}$. These facts contrast starkly with the lack of awareness of the population and health authorities on this topic.

The economic burden of the diagnostic and therapeutic management of these chronic pathologies has jeopardised the economic sustainability of healthcare systems, especially in the case of public systems with universal coverage. To deal with this problem it is absolutely necessary to rationalise spending if we want to avoid inequity of access to healthcare services.
TABLE 3. Future challenges for healthcare systems

- Guaranteeing economic sustainability of the system

- Rationalisation of spending versus inequity

- Change in mentality of citizens: move from the "right to health" to the "obligation to care for oneself"

- Patient empowerment: increase their participation in decision-making

- More demanding systems for evaluating cost-efficiency

- Personalised health management

- Rational management of chronicity: - Early diagnosis and avoiding under-diagnosis

- Improve the coordination of assistive services

- Utilizing integral solutions: product + service + education

- Increase of healthcare activity in the patient's home

- End-of-life management

- Implementation of "digital health"

- Accelerate the translation/transfer of results from research to care

- Redefinition of the doctor's role; integration with other health professionals: paramedics, health agents, statisticians

- Adapt teaching of medicine and other health professions to new needs

- Global strategies to avoid global pandemics

In order for health costs to be affordable, a shift in mentality in a population for whom health has been considered a right will be essential. Citizens should be aware of the enormous economic burden borne by healthcare administrations and the need to collaborate on their own wellbeing based on the moral obligation to take care of their health, avoiding as much as possible those circumstances that undermine it. Under this perspective of responsibility, citizens should increase their participation in the establishment of priorities and decision-making by healthcare officials.

Faced with the challenge of sustainability, healthcare administrations have opted to put in place more demanding evaluation systems that allow selecting those solutions with a higher level of cost-efficiency and to evaluate in a rational way new alternatives. Another potentially useful strategy would be to develop a stricter definition of criteria that allow for a more personalised therapeutic approach 
allowing resources to be allocated in those cases with the greatest expectation of success.

A large portion of health resources is allocated to the care of chronic diseases. However, when analysing the functioning of health systems in the management of chronic diseases, it is evident that there is a lack of coordination among resources: primary care, specialised care, hospitalisation, convalescence and social facilities. In general, the existence of under-diagnosis of chronic diseases is confirmed so many patients suffer without being diagnosed or they are diagnosed when the disease has significantly evolved and to a large extent the window of opportunity for an effective treatment has disappeared.

When analysing the configuration of healthcare resources, it is frequently observed that in practice, chronic diseases are attended by services designed to treat acute conditions. Progress is needed in the design of services specifically tailored for the care of chronic pathologies that coordinate all the actors, avoiding duplications and sharing all information about a specific case and counting on integral solutions (product + service + education). On the other hand, it could be very useful to design services as an alternative to hospitalisation, through the increase of care activity at home and possibly with the help of modern remote monitoring technologies, an option which should be the subject of a thorough evaluation. Finally, a redefinition of the care strategies for the final period of life is mandatory, where a large part of the health cost is often concentrated without a tangible benefit in terms of quality of life.

Recent technological developments derived from digitalisation are allowing the progressive implementation of what is known as digital health that will revolutionise healthcare systems in a profound way. One example of such potentialities is the International Business Machines (IBM) Watson Health initiative. All these changes are taking place at a much greater speed than that allowed by the current medical practice. Therefore, it will be absolutely necessary to rethink the way in which knowledge transfer from research to care occurs. In any case, it is evident that the mode of action of health personnel will change completely and that physicians must integrate their activity with advanced artificial intelligence systems and share their role with that of other health professionals such as para-medicals, health agents, software developers, statisticians, and so on. To enable this, the way in which the teaching of the health professions is carried out must be radically modified.

Finally, depending on existing threats, it is expected that it will be necessary to design a response strategy organised at a global level that allows the risk of a mega-pandemic to be solved in a sustainable manner.

\section{FUTURE BIOMEDICAL RESEARCH TRENDS IN RESPIRATORY MEDICINE}

In the past decades, several large-scale local and international research studies in respiratory diseases led to remarkable life-changing results in diseases such as asthma, sleep apnoea, cystic fibrosis and pulmonary hypertension, among others. Lung transplantation has notably improved its rate of success and new treatments have been developed to treat idiopathic pulmonary fibrosis (IPF). Nevertheless, despite these evident successes, there are many gaps that need to be effectively addressed. 
For this purpose, there is an evident need to substantially increase the national and international, public and private, investments to support research in respiratory diseases the number of which is far below an appropriate percentage when comparing prevalence and mortality. As an example, despite the high prevalence of respiratory diseases, only $4.3 \%$ of the health budget of the Seventh Framework Programme for Research and Technological Development of the European Union was allocated to respiratory research ${ }^{6}$. Respiratory medical research has been shown to represent a six-fold return on investment ${ }^{7}$.

Participants in the meeting thought that biomedical research in respiratory medicine should concentrate on the following areas, listed in table 4:

\section{1 - P4 Medicine}

Traditional medical practice has been historically "reactive". This is, physicians act when there is a medical problem (i.e., a disease) and try to help the (already so-called) patient to cure or, at least, improve her/his condition. Due to several technological advances (including high throughput "omic" technologies, imaging and bioinformatics, among others), a major change in clinical care is anticipated to occur in the near future; in fact, it is already occurring in some places. A popular term to describe this change is "P4 medicine" (others are personalised, individualised or precision medicine) $)^{8-14}$. The acronym P4 medicine stems from the fact that this strategy sought to promote and preserve health before "the problem" arises and is based on four different components (or "P's"): 1) Personalised, which indicates that the strategy is based on the
TABLE 4. Future biomedical research trends in respiratory medicine

1. Predictive, Preventive, Personalised and Participatory (4P) Medicine

2. Big data \& systems biology

3. Biomarkers \& biological monitoring

4. Early origins of lung diseases

5. Imaging technologies

6. Microbiome

7. New therapeutic approaches

8. Health information technologies (e-health)

unique molecular background that each person has; 2) Predictive, because (at least theoretically) the knowledge of each individual genetic background may allow the prediction of future risk(s) of disease(s); 3) Preventive, because (again in theory) knowing the risks offer opportunities for prevention; and, finally, 4) Participative, because the previous three " $\mathrm{P}$ 's" require the consent and active participation of the patient. The development of P4 medicine offers (again in theory) great opportunities and may really be a truly transforming element that can radically change the way health care systems operate today. However, it also associated with great uncertainties, including ethical, economic, logistic, technological, social, and equity elements, among others ${ }^{8-14}$.

\section{2 - Big data and systems biology}

The so-called Moore law states that overall computing processing power doubles every two years. This extraordinary evolution has opened unforeseen opportunities in everyday life (personal computers, mobile phones, internet, and many more) and, of course, in medical practice and research ${ }^{15}$. For instance, in clinical practice, all modern imaging techniques (including echo, computed tomography $[\mathrm{CT}]$ and magnetic 
resonance imaging [MRI]) would not be possible without computers ${ }^{15}$. In research, big data analysis and systems biology are two paradigmatic examples. The former refers to the capacity to analyse, literally, millions of data points in any biomedical research field ${ }^{16-18}$. Computing power is not the problem. The problem is the quality of the database analysed, which is often incomplete and non-specific. The current development of electronic medical health records will likely improve this situation and contribute to facilitate the use of the huge repository of relevant medical data that is daily generated in clinical practice around the world ${ }^{19}$. On the other hand, systems biology (and its medical equivalent, network medicine) is a novel research strategy that tries to address the complexity of any biological system (including the human body) ${ }^{20-25}$. Diseases rarely occur because a single element of the system fails (e.g., one gene). On the contrary, all molecules (deoxyribonucleic acid [DNA], ribonucleic acid [RNA], proteins, lipids), cells and organs form a very complex and inter-related system. We need a holistic approach to it, like the one offered by systems biology and network medicine, to understand it and to modify it in order to preserve (or eventually regain) health ${ }^{20-25}$.

\section{3 - Biomarkers and biological monitoring}

Genomics, proteomics and metabolomics are new tools with a great potential in the diagnosis and control of diverse respiratory diseases and provide elements to define personalised therapeutic approaches (Fig. 3). Genomic studies are currently being used for the diagnosis of cystic fibrosis, several neuromuscular diseases

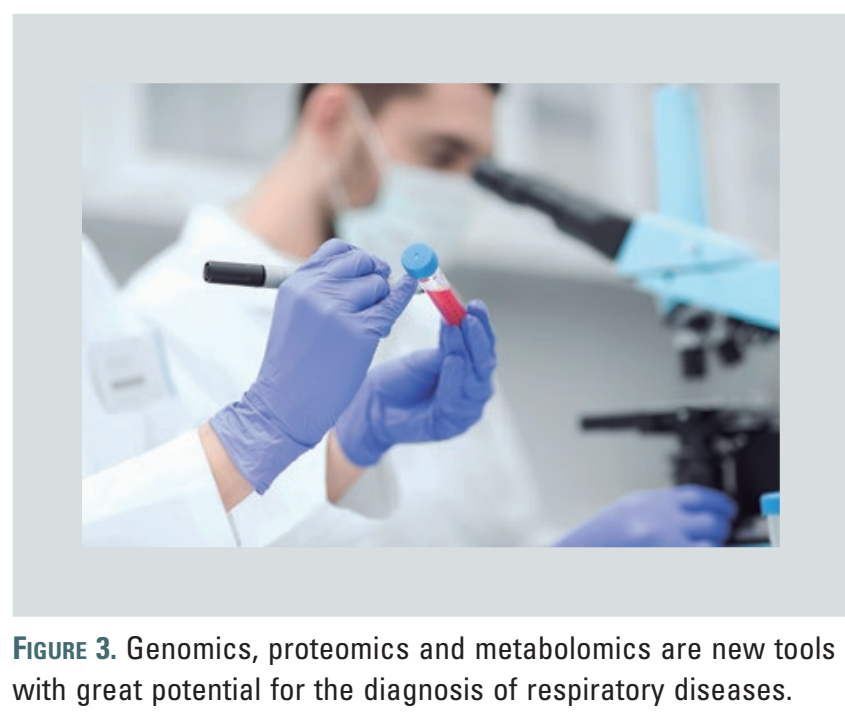

and some rare diseases, but there is a huge potential for determining the individual predisposition to different kinds of malignancies. Blood microRNAs have been developed for the diagnosis of cancer, certain infections and some rare diseases ${ }^{26,27}$. On the other hand, epigenetic techniques will be determinant for establishing the influence of the environment on the expression of some gene disturbances. Finally, telomere length may serve as a surrogate marker for the identification of patients at greatest risk for carrying mutant telomerase genes. These mutations have been related to the development of certain respiratory diseases such as idiopathic pulmonary fibrosis and $\mathrm{COPD}^{28}$. Therefore, telomere shortening studies may potentially heighten the index of suspicion of such conditions and facilitate diagnosis.

Detection of exhaled volatile compounds may be very useful in the screening of certain malignancies and in the follow-up of inflammatory lung diseases ${ }^{29}$. Proteomic and metabolomic techniques of breath condensate have a promising role in the diagnosis and control of 
different lung inflammatory diseases ${ }^{30}$. Finally, genomic techniques such as deep sequencing permit a precise characterisation of both known and novel pathogens to control the emergence of resistance to prescribed treatments ${ }^{31}$.

\section{4 - Early origins of lung diseases}

The traditional understanding of human adult diseases is that they are due to the interaction of environmental and genetic factors during adulthood and geriatric years. Recently, a new vision is emerging that states that early life events (both before and after birth) can have a significant impact in adult health later in life. Baker was the first investigator who indicated this in $1991^{32}$. Since then, a number of studies have consolidated this view ${ }^{33-35}$. For instance, a recent study has shown that between $4-13 \%$ of the general population have sub-optimally developed lungs and that this is very likely accompanied by an abnormal development of other organ systems, such as the cardiovascular or metabolic ones ${ }^{36}$. This results in a higher prevalence and presentation of comorbidities about a decade earlier as well as in premature death ${ }^{36}$. All in all, these observations open new questions for research but, very importantly, novel opportunities for the prevention and earlier treatment of chronic diseases in the adults and elderly ${ }^{37}$.

\section{5 - Imaging technologies}

Imaging technologies already play a fundamental role in the management of respiratory diseases and they are constantly improving. New imaging techniques have a tremendous potential in chest medicine. Real-time MRIs allow pathophysiological assessment in pulmonary hypertension ${ }^{38}$. Three-dimensional ultra-sonographic computing has a great potential in the study of different kinds of respiratory diseases such as vascular pulmonary abnormalities, pulmonary hypertension as well as in the characterisation of pleural diseases ${ }^{39}$. Another alternative is three-dimensional CT with great potential in the study of different kinds of chronic respiratory diseases such as COPD, asth$\mathrm{ma}$, interstitial lung diseases and also lung can$\mathrm{cer}^{40}$. A different type of approach is metabolic imaging through improved positron emission tomography (PET) with potential applications in thoracic cancer as well as in inflammatory diseases ${ }^{41}$. Finally, there is a great potential in the use of nanotechnology to target "in vivo" tumoural or inflammatory cells for imaging purposes.

Airways imaging has made a significant progress through the introduction of auto-fluorescence bronchoscopy, confocal laser micro-endoscope, and optical coherence tomography $(\mathrm{OCT})^{42-44}$. Confocal microscopy permits an "in vivo" assessment of vaso-activity phenomena during hypoxia, ischaemia-reperfusion events in inflammatory diseases and cancer ${ }^{45}$. Electromagnetic navigation bronchoscopy (ENB) permits a minimally invasive approach to access difficult-to-reach areas of the lung ${ }^{46}$.

\section{6 - Microbiome}

The introduction of culture-independent techniques for the microbiological analysis of respiratory samples has confirmed that the respiratory system hosts a large number of microorganisms. Microbiome analyses of the 
bacterial composition of respiratory samples have been based on the gene encoding $16 \mathrm{~S}$ ribosomal RNA (16S rRNA) and have shown that only $1 \%$ of the bacteria part of the respiratory microbiome is recovered from cultures. Sequencing of the $16 \mathrm{~S}$ rRNA gene from respiratory samples allows a taxonomic classification of each bacterium identified and is used to describe the composition of a microbial ecosystem as a whole ${ }^{47}$. Reference databases of the 16S rRNA gene allow to classify the sequences in the sample from the highest to the lowest taxonomic levels (phylum and genus, respectively), in most cases reaching also the species level.

In the healthy subject the microbial flora of the respiratory tree is phylogenetically diverse $^{48,49}$, with Firmicutes, Bacteroidetes and Proteobacteria as their most frequent phyla, and a low frequency of operational taxonomic unit (OTU)s corresponding to potentially pathogenic microorganisms ${ }^{50}$. The microbiome harboured by the bronchial tree and the oropharynx have a close similarity in the healthy subject, mainly due to aspiration of oro-pharyngeal secretions during sleep ${ }^{51,52}$. The local environment of the bronchial tree and the lung is able to modify the microbiome after the aspiration of secretions, however, as shown by the higher relative abundance of some bacteria part of the Prevotella genus respiratory samples ${ }^{52}$.

In most chronic respiratory diseases microbiome analyses of sputum samples have demonstrated an important decline in bacterial diversity, with a change to a restricted flora which include most of the bacteria commonly considered as potentially pathogenic microorganisms, paralleled by a decline in the relative abundance of other bacteria. The presence of a restricted number of genera attaining high relative abundance in chronic respiratory diseases is pattern that is considered as dysbiosis, and potentially related to harmful effects.

Interaction between different microorganisms can be addressed through microbiome analyses, and functional meta-genomics, an approach that describes a genomic potential of a community, sometimes show clear changes in specific pathways, although the bronchial microbiome as a whole may not have changed significantly ${ }^{53}$. These functional changes are potentially important because through them the resident community as a whole shows its power to modify important metabolic patterns without changing significantly in its composition.

Although the microbial composition of the respiratory flora is being known through microbiome analyses, the involvement of the respiratory flora in the pathogenesis of respiratory diseases, and especially the role microorganisms considered non-pathogenic by culture-based microbiology, is practically unknown. Bacterial diversity loss, often related to an increase in the relative abundance of specific phyla as Proteobacteria, is associated with advanced disease, and may be one of the determinants which influences its progression. Meta-genomics and the analysis of bacterial RNA will be able to provide functional information on the respiratory microbiome, detailing interactions between viruses, fungi and bacteria, and, potentially, to facilitating the design of intervention studies aimed at conserving the flora that acts as positive mutualist, as opposed to the respiratory 
pathogens that progressively replace it when COPD progresses to advanced disease.

\section{7 - New therapeutical approaches}

So-called "biological treatments" use substances obtained totally or in part from living organisms capable to stimulate or suppress the ability of the body's immune system to fight diseases such as cancer, infection, or certain inflammatory diseases. Types of biological therapy include immunotherapy (vaccines, cytokines, and antibodies), gene therapy, and some targeted therapies. Biological therapy often involves the use of substances called biological response modifiers (BRMs) normally produced by de body in small amounts in response to infection and disease. Thanks to modern laboratory techniques BRMs, such as blocking antibodies, antagonists against receptors or inflammatory enzyme inhibitors can be produced in large amounts for use in the treatment of cancer and diverse inflammatory diseases.

During recent years, biological treatments have been developed to treat different respiratory diseases such as asthma, COPD, IPF, pulmonary hypertension and lung cancer ${ }^{54-57}$ according to a "personalised" therapeutical context. Regenerative medicine is another promising approach for several severe diffuse pulmonary diseases, such as IPF o acute respiratory distress syndrome (ARDS) through basic research on the cellular and molecular properties of stem cells ${ }^{58}$. Gene therapy is another novel strategy with great potential in the treatment of diseases such as cystic fibrosis where the gene encoding a protein called the cystic fibrosis trans-membrane conductance regulator (CFTR) is faulty. The delivery of a correct copy of the faulty CFTR gene to cells in the lungs may be an effective treatment. This approach has already been used in several studies with limited evidence of benefit ${ }^{59}$.

Other new strategies such as the clustered regularly interspaced short palindromic repeat (CRISPR) technology, have been used to rapidly, easily and efficiently modify endogenous genes in a wide variety of biomedically important cell types leading to perform targeted, highly efficient modifications of genome sequence and gene expression with great potential in the development of novel molecular therapeutics for human disease ${ }^{60}$.

Finally, there are new technologies such as artificial lungs that have been developed to treat acute respiratory failure or as a bridge to lung transplantation in chronic respiratory failure $^{61}$. Worth mentioning are also novel endoscopic approaches intended to perform lung volume reduction in severe emphyse$\mathrm{ma}^{62}$ or thermoplasty in severe asthma ${ }^{63}$.

\section{8 - Health information technologies (e-health)}

Health information technologies (HITs) have a tremendous potential for becoming routine tools for remote monitoring of patients suffering from chronic respiratory diseases or assisting them in self-monitoring and decision-making. This approach will be of great interest considering the increasing percentage of population suffering from chronic illness and the limited public resources to provide care ${ }^{64}$. Relatively simple technologies such as certain web applications and smartphones have been introduced to monitor respiratory diseases such as asthma or 
COPD at home prompting the use of therapeutic measures and better understanding of the environmental triggers ${ }^{65}$.

\section{CONCLUSIONS}

In recent decades there has been an accelerated change of multiple factors which directly or indirectly affect living conditions for modern society. These changes will have an impact on human health as well as on the way in which health systems and individuals will respond to health risks. Considering the specific case of respiratory medicine all these phenomena result in a higher incidence of pathologies related to environmental changes, more precisely the deterioration of air quality. Chronic respiratory diseases are not only already very common, but their prevalence appears to be increasing and related mortality remains quite high. Respiratory diseases present a great challenge to human health and a productive economy. Prevention, control and cure of these conditions are among the most cost-effective interventions to be taken and must be a priority for researchers as well as for the health authorities.

Nowadays, the future of respiratory medicine looks very promising. Thus, many novel technologies substantially improve imaging and biological monitoring through the different "omics" has great potential in the diagnosis and control of multiple diseases allowing to personalised therapeutic approaches, which is far more effective and causes fewer side effects. In order to facilitate the rapid implementation of these capabilities there is a pressing need to substantially increase the national and international, public and private, investments to support research in respiratory diseases which receive well below their value when considering impact in terms of prevalence and mortality.

\section{ACKNOWLEDGEMENTS}

To Joan Casals PhD (Momentum) for his logistical support in organizing the BRN Retreat and in summarising the main conclusions.

\section{CONFLICTS OF INTERESTS}

Dr. Jordi Dorca, Dr. Alvar Agustí and Dr. Eduard Monsó have nothing to disclose in relation to the submitted work.

\section{APPENDIX}

\section{List of participants}

Jorge Abad (Hospital Germans Trias i Pujol), Àlvar Agustí (Hospital Clínic), Noemí Arnau (Ferrer), David Asín (Linde), Angel Francisco Azpeitia (Esteve), Pablo Calzada (Aldo Unión), Jordi Dorca (Hospital de Bellvitge), Joan Escarrabill (Pla Director Malalties Aparell Respiratori), Rosa Faner (Hospital Clínic), Andrés Fernández (Ferrer), Carlos Fina (Esteve Teijin), Joaquim Gea (Hospital del Mar), Gonzalo de Miquel (Astra Zeneca), Jaime Grego (Leti), Eduard Monsó (Hospital del Parc Taulí), Eusebi Ocaña (Esteve Teijin), Vicente Plaza (Hospital de Sant Pau), Robert Rodriguez-Roisin (BRN Reviews), Antoni Rosell (Hospital de Bellvitge), Joan Ruiz (Hospital Germans Trias i Pujol) and Alfons Torrego (Hospital de Sant Pau). 


\section{REFERENCES}

1. GBD 2015 Chronic Respiratory Disease Collaborators. Global, regional, and national deaths, prevalence, disability-adjusted life years, and years lived with disability for chronic obstructive pulmonary disease and asthma, 1990-2015: a systematic analysis for the Global Burden of Disease Study 2015. Lancet Respir Med. 2017;5:691-706.

2. Institute for Health Metrics and Evaluation (IHME). GBD Compare Data Visualization. Seattle, WA: IHME, University of Washington; 2017. Available from: https://vizhub.healthdata.org/gbd-compare/.

3. Marciniuk D, Ferkol T, Nana A et al. Respiratory diseases in the world: Realities of today - opportunities for tomorrow. Forum of International Respiratory Societies. Switzerland. European Respiratory Society, 2013.

4. GBD 2015 Mortality and Causes of Death Collaborators. Global, regional, and national life expectancy, all-cause mortality and cause-specific mortality for 249 causes of death, 1980-2015: a systematic analysis for the Global Burden of Disease Study 2015. Lancet 2016;388: 1459-1544.

5. Burney PG, Patel J, Newson R, Minelli C, Naghavi M. Global and regional trends in COPD mortality, 1990- 2010. Eur Respir J. 2015;45:1239-47.

6. Gibson GJ, Loddenkemper R, Sibille Y et al. European Lung White Book. 2nd edition. Sheffield: European Respiratory Society. 2013;38:414-23.

7. The Australia Society for Medical Research. The value of investing in health R\&D in Australia II. Sydney. The Australia Society for Medical Research, 2007.

8. Galas DJ, Hood L. Systems biology and emerging technologies will catalyze the transition from reactive medicine to predictive, personalized, preventive and participatory (P4) medicine. IBC. 2009;1:1-4

9. Hood L, Balling R, Auffray C. Revolutionizing medicine in the 21(st) century through systems approaches. Biotechnol J. 2012;7:10.

10. Agusti A, Sobradillo P, Celli B. Addressing the Complexity of Chronic Obstructive Pulmonary Disease: From Phenotypes and Biomarkers to ScaleFree Networks, Systems Biology, and P4 Medicine. Am J Respir Crit Care Med. 2011; 183:1129-37.

11. König IR, Fuchs O, Hansen G, von Mutius E, Kopp MV. What is precision medicine? Eur Respir J. 2017;50.

12. Bahcall O. Precision medicine. Nature. 2015;526:335.

13. Aronson SJ, Rehm HL. Building the foundation for genomics in precision medicine. Nature. 2015;526:336-42.

14. Schork NJ. Personalized medicine: Time for one-person trials. Nature. 2015; 520: 609-11.

15. Agustí A. Predicting the future from the past. Eur Respir J. 2017;49.

16. Chai S, Shih W. Why Big Data isn't enough. MIT Sloan Management Review. 2017; 58:57-61.

17. Auffray C, Balling R, Barroso I et al. Making sense of big data in health research: Towards an EU action plan. Genome Med. 2016;8:71.

18. Obermeyer Z, Emanuel EJ. Predicting the Future - Big Data, Machine Learning, and Clinical Medicine. New England Journal of Medicine. 2016; 375:1216-9.

19. Frankovich J, Longhurst CA, Sutherland SM. Evidence-Based Medicine in the EMR Era. New England Journal of Medicine. 2011;365:1758-9.

20. Kitano H. Systems Biology: A Brief Overview. Science. 2002;295:1662-4.

21. Noell G, Faner R, Agusti A. From systems biology to P4 medicine: applications in respiratory medicine. Eur Respir Rev 2018;27.

22. Thamrin C, Frey U, Kaminsky DA, Reddel HK, Seely AJE, Suki B, Sterk PJ. Systems Biology and Clinical Practice in Respiratory Medicine: The Twain Shall Meet. Am J Respir Crit Care Med. 2016; 194:1053-61.

23. Menche J, Sharma A, Kitsak M, et al. Disease networks. Uncovering disease-disease relationships through the incomplete interactome. Science. 2015;347:1257601.

24. Agusti A, Lopez-Giraldo A, Cruz T, Faner R. Relevance of systems biology to respiratory medicine. BRN Rev. 2016;2:1-13.

25. Barabasi AL, Gulbahce N, Loscalzo J. Network medicine: a network-based approach to human disease. Nat Rev Genet. 2011; 12: 56-68.
26. Wheelock CE, Goss VM et al. Application of 'omics technologies to biomarker discovery in inflammatory lung diseases. Eur Resp J. 2013;42: 802-26.

27. Narita M, Shimura E, Nagasawa A et al. Chronic treatment of non-small-cell lung cancer cells with gefitinib leads to an epigenetic loss of epithelial properties associated with reductions in microRNA-155 and -200c. PLoS One. 2017; 12:e0172115.

28. Snetselaar R, van Batenburg AA, van Oosterhout MFM et al. Short telomere length in IPF lung associates with fibrotic lesions and predicts survival. PLoS One. 2017;7;12:e0189467.

29. Nardi-Agmon I, Peled N. Exhaled breath analysis for the early detection of lung cancer: recent developments and future prospects. Lung Cancer (Auckl). 2017;17;8:31-8.

30. Andrianjafimasy M, Zerimech F, Akiki Z et al. Oxidative stress biomarkers and asthma characteristics in adults of the EGEA study. Eur Respir J 2017;28;50. pii: 1701193.

31. Prachayangprecha S, Schapendonk CM, Koopmans MP et al. Exploring the potential of next-generation sequencing in detection of respiratory viruses. J Clin Microbiol. 2014; 52:3722-30.

32. Barker DJ. The fetal and infant origins of adult disease. BMJ. 1990;301:1111

33. Lange $\mathrm{P}$, Celli B, Agusti A et al. Lung-Function Trajectories Leading to Chronic Obstructive Pulmonary Disease. N Engl J Med. 2015;373:111-22.

34. Martinez FD. The Origins of Asthma and Chronic Obstructive Pulmonary Disease in Ea rly Life. Proc Am Thorac Soc. 2009;6:272-7.

35. Martinez FD. Early-Life Origins of Chronic Obstructive Pulmonary Disease. N Engl J Med. 2016;375:871-8.

36. Agusti A, Faner R. Beyond smoking: new paradigm, novel opportunities. Lancet Respir Med. 2018;6:324-32.

37. Agustí A, Noell G, Brugada J, Faner R. Lung function in early adulthood and health in later life: a transgenerational cohort analysis. Lancet Respir Med. 2017;5:935-45.

38. Barber NJ, Ako EO, Kowalik GT et al. Magnetic Resonance-Augmented Cardiopulmonary Exercise Testing: Comprehensively Assessing Exercise Intolerance in Children With Cardiovascular Disease. Circ Cardiovasc Imaging. 2016; 9:pii: e005282.

39. Raza F, Dillane C, Mirza A et al. Differences in right ventricular morphology, not function, indicate the nature of increased afterload in pulmonary hypertensive subjects with normal left ventricular function. Echocardiography. 2017; 34:1584-92.

40. Zhang L, Li M, Li Z, Kedeer X, et al. Three-dimensional printing of navigational template in localization of pulmonary nodule: A pilot study. J Thorac Cardiovasc Surg. 2017; 154:2113-9.

41. Greenspan BS. Role of PET/CT for precision medicine in lung cancer perspective of the Society of Nuclear Medicine and Molecular Imaging. Transl Lung Cancer Res. 2017;6:617-20.

42. Yserbyt J, Dooms C, Janssens W et al. Endoscopic advanced imaging of the respiratory tract: exploring probe-based confocal laser endomicroscopy in emphysema. Thorax. 2018;73:188-90.

43. Andolfi M, Potenza R, Capozzi R et al. The role of bronchoscopy in the diagnosis of early lung cancer: a review. J Thorac Dis. 2016; 8:3329-37.

44. Zhang J, Wu J, Yang Y et al. White light, autofluorescence and narrow-band imaging bronchoscopy for diagnosing airway pre-cancerous and early cancer lesions: a systematic review and meta-analysis. J Thorac Dis. 2016 8:3205-16.

45. Kobayashi $\mathrm{Y}$, Uehara $\mathrm{T}$, Kawasaki $\mathrm{K}$ et al. Three-dimensional analysis of alveolar wall destruction in the early stage of pulmonary emphysema. Clin Anat. 2015;28:227-34.

46. Brown J, Lee TJ, Joiner T et al. Using Electromagnetic Navigation Bronchoscopy and Dye Injection to Aid in Video-Assisted Lung Resection. Am Surg. 2016; 82:1052-4.

47. Woese CR, Kandler O, Wheelis ML. Towards a natural system of organisms: proposal for the domains Archaea, Bacteria, and Eucarya. Proc Natl Acad Sci U S A; 1990;87:4576-9.

48. Erb-Downward JR, Thompson DL, Han MK et al. Analysis of the lung microbiome in the "healthy" smoker and in COPD. PLoS One. 2011;6:e16384. 
49. Charlson ES, Bittinger K, Chen J et al. Assessing bacterial populations in the lung by replicate analysis of samples from the upper and lower respiratory tracts. PLoS One. 2012;7:e42786.

50. Charlson ES, Bittinger K, Haas AR et al. Topographical continuity of bacterial populations in the healthy human respiratory tract. Am J Respir Crit Care Med. 2011;184:957-63.

51. Dickson RP, Erb-Downward JR, Freeman CM et al. Spatial Variation in the Healthy Human Lung Microbiome and the Adapted Island Model of Lung Biogeography. Ann Am Thorac Soc. 2015;12:821-30.

52. Bassis CM, Erb-Downward JR, Dickson RP et al. Analysis of the upper respiratory tract microbiotas as the source of the lung and gastric microbiotas in healthy individuals. MBio. 2015;6:e0037.

53. Millares L, Pérez Brocal V, Ferrari R et al. Functional Metagenomics of the Bronchial Microbiome in COPD. PloS One. 2015;10:e0144448.

54. Nachef Z, Krishnan A, Mashtare T et al. Omalizumab versus Mepolizumab as add-on therapy in asthma patients not well controlled on at least an inhaled corticosteroid: A network meta-analysis. J Asthma. 2017; $1: 1-12$.

55. Sulaiman I, Lim JC, Soo HL, Stanslas J. Molecularly targeted therapies for asthma: Current development, challenges and potential clinical translation. Pulm Pharmacol Ther. 2016; 40:52-68.

56. Wang FP, Liu T, Lan Z et al. Efficacy and Safety of Anti-Interleukin-5 Therapy in Patients with Asthma: A Systematic Review and Meta-Analysis. PLoS One. 201611:e0166833.
57. Pavord ID, Chanez P, Criner GJ et al. Mepolizumab for Eosinophilic Chronic Obstructive Pulmonary Disease. N Engl J Med. 2017; 377:1613-29.

58. Cárdenes N, Cáceres E, Romagnoli M, Rojas M. Mesenchymal stem cells: a promising therapy for the acute respiratory distress syndrome. Respiration. 2013;85:267-78

59. Perry LA, Penny-Dimri JC, Aslam AA, Lee TWR, Southern KW. Topical cystic fibrosis transmembrane conductance regulator gene replacement for cystic fibrosis-related lung disease. Cochrane Database Syst Rev. 2016; CD005599.

60. Sander JD, Joung JK. CRISPR-Cas systems for genome editing, regulation and targeting. Nat Biotech. 2014;32:347-55.

61. Morelli A, Del Sorbo L, Pesenti A et al. Extracorporeal carbon dioxide removal (ECCO2R) in patients with acute respiratory failure. Intensive Care Med. 2017;43:519-30.

62. Slebos DJ, Shah PL, Herth FJ et al. Endobronchial Valves for Endoscopic Lung Volume Reduction: Best Practice Recommendations from Expert Panel on Endoscopic Lung Volume Reduction. Respiration. 2017;93:138-50.

63. Trivedi A, Pavord ID, Castro M. Bronchial thermoplasty and biological therapy as targeted treatments for severe uncontrolled asthma. Lancet Respir Med. 2016;4:585-92.

64. Himes BE and Weitzman E. Innovations in health information technologies for chronic pulmonary diseases. Respir Res. 2016;17:38.

65. Trivedi D. Cochrane review summary: smartphone and tablet self-management apps for asthma. Prim Health Care Res Dev. 2015;16:111-9. 